\title{
TEORIA DA ADAPTAÇÃO E SAÚDE DO TRABALHADOR EM HOME OFFICE NA PANDEMIA DE COVID-19
}

\section{THEORY OF ADAPTATION AND WORKER'S HEALTH IN HOME OFFICE IN THE COVID-19 PANDEMIC}

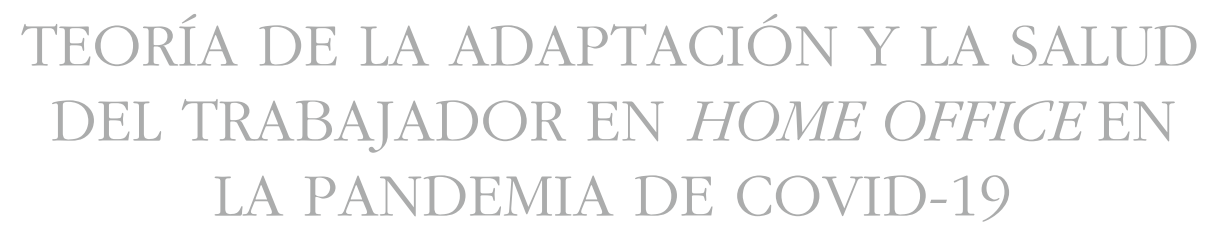

Nathalia Henriques Veiga

Yan Zi Li Figueiredo Ten ${ }^{2}$

Vithória Paes Machado ${ }^{3}$

Magda Guimarães de Araújo Faria ${ }^{4}$

Mercedes de Oliveira Neto ${ }^{5}$

Helena Maria Scherlowski Leal David

Como citar este artigo: Veiga NH, Ten YZLF, Machado VP, Faria MGA, Oliveira Neto M, David HMSL. Teoria da adaptação e saúde do trabalhador em Home Office na pandemia de COVID-19. Rev baiana enferm. 2021;35:e37636.

Objetivo: refletir sobre as implicações do trabalho em Home Office no período da pandemia de COVID-19 na saúde dos indivíduos, sob a perspectiva da Teoria da Adaptação desenvolvida por Callista Roy. Método: estudo reflexivo baseado na aplicação da Teoria da Adaptação desenvolvida por Callista Roy relacionada às modificações do processo de trabalho impostas pela crise sanitária da pandemia de COVID-19, com ênfase no Home Office. Resultados: a Teoria da Adaptação de Callista Roy possui quatro modos adaptativos: fisiológico, autoconceito, desempenho de papel e interdependência. É possível verificar a interlocução de todas essas dimensões no trabalho em Home Office imposto pelo contexto da pandemia. Conclusão: a Teoria de Callista Roy subsidia as discussões sobre a possibilidade de adaptação neste novo contexto, seja de maneira pontual ou mediante transformações no processo de trabalho em longo prazo, superando limitações do indivíduo e descobrindo maneiras de se fazer e ser no campo do trabalho.

Descritores: Saúde do Trabalhador. Teoria de Enfermagem. Enfermagem. Pandemias. Infecções por Coronavírus.

Objective: to reflect on the implications of Home Office work in the covid-19 pandemic period on individuals' health, from the perspective of the Adaptation Theory developed by Callista Roy. Method: reflective study based on the application of the Adaptation Theory developed by Callista Roy related to changes in the work process imposed by the health crisis of the COVID-19 pandemic, with emphasis on the Home Office. Results: Callista Roy's Adaptation Theory has four adaptive modes: physiological, self-concept, role performance and interdependence. There is the interlocution of all these dimensions at Home Office work imposed by the pandemic context. Conclusion: Callista

\footnotetext{
Estudante de Enfermagem. Universidade Estadual do Rio de Janeiro. Rio de Janeiro, Rio de Janeiro, Brasil. nathaliahveiga@gmail.com. http://orcid.org/0000-00034348-07I3.

Estudante de Enfermagem. Universidade Estadual do Rio de Janeiro. Rio de Janeiro, Rio de Janeiro, Brasil. http://orcid.org/0000-0002-8587-6460.

Estudante de Enfermagem. Universidade Estadual do Rio de Janeiro. Rio de Janeiro, Rio de Janeiro, Brasil. https://orcid.org/0000-0003-3345-3367.

Enfermeira. Doutora em Enfermagem. Professora da Universidade Estadual do Rio de Janeiro. Rio de Janeiro, Rio de Janeiro, Brasil. http://orcid.org/0000-000 I9928-6392.

5 Enfermeira. Doutora em Enfermagem. Professora da Universidade Estadual do Rio de Janeiro. Rio de Janeiro, Rio de Janeiro, Brasil. http://orcid.org/0000-000 I7529-9535.

6 Enfermeira. Doutora em Saúde Pública. Professora da Universidade Estadual do Rio de Janeiro. Rio de Janeiro, Rio de Janeiro, Brasil. http://orcid.org/0000-000 I8002-6830.
} 
Roy's Theory supports discussions about the possibility of adaptation in this new context, either in a specific way or through transformations in the long-term work process, overcoming limitations of the individual and discovering ways to do and be in the work field.

Descriptors: Worker's Health. Nursing Theory. Nursing. Pandemics. Coronavirus Infections.

Objetivo: reflexionar sobre las implicaciones del trabajo Home Office en el período de la pandemia de COVID-19 sobre la salud de las personas, desde la perspectiva de la Teoría de la Adaptación desarrollada por Callista Roy. Método: estudio reflexivo basado en la aplicación de la Teoría de la Adaptación desarrollada por Callista Roy relacionado con cambios en el proceso de trabajo impuestos por la crisis de salud de la pandemia de COVID-19, con énfasis en el Home Office. Resultados: La Teoría de la Adaptación de Callista Roy tiene cuatro modos adaptativos: fisiológico, autoconcepto, ejecución del papel e interdependencia. Puede verificarse la interlocución de todas estas dimensiones en el trabajo Home Office impuesto por el contexto de la pandemia. Conclusión: la Teoría de Callista Roy apoya discusiones sobre la posibilidad de adaptación en este nuevo contexto, ya sea de manera puntual o a través de transformaciones en el proceso de trabajo a largo plazo, superando las limitaciones del individuo y descubriendo formas de hacer y estar en el campo del trabajo.

Descriptores: Salud del Trabajador. Teoría de Enfermería. Enfermería. Pandemias. Infecciones por Coronavirus.

\section{INTRODUÇÃO}

Covid-19 foi o nome criado para representar o conjunto de sintomas provocado pela infecção pelo novo coronavírus, o SARS-CoV-2, o qual afeta essencialmente o sistema respiratório ${ }^{(1-2)}$. O avançar da doença culminou na disseminação mundial e acabou sendo conhecida como a primeira pandemia da história provocada por um coronavírus, anunciada no dia 11 de março de $2020^{(3)}$.

Como resposta do atual governo federal, a Lei $\mathrm{n}^{\mathrm{o}} 13.979 / 20^{(4)}$ foi criada para firmar as medidas da emergência de saúde pública ao enfrentamento do novo coronavírus, entre elas o isolamento, a quarentena, a realização compulsória de exames médicos, testes laboratoriais etc. Portanto, mediante a implementação dessas medidas, principalmente do isolamento social, o trabalhador brasileiro precisou reinventar seu processo de trabalho, muitas vezes precisando trabalhar em casa, na modalidade Home Office.

O Home Office caracteriza-se pelo desempenho das atividades profissionais no mesmo ambiente em que se reside ${ }^{(5)}$. Nessa perspectiva, essa modalidade tornou-se estratégia viável para a manutenção de atividades laborais relacionados a processos de trabalho compatíveis. Apesar de não haver dados que indiquem o percentual de indivíduos atuando em Home Office, as taxas de isolamento fornecidas pela geolocalização dos aparelhos telefônicos indicam que 48,9\% da população brasileira estava em isolamento no dia 14 de junho de 2020, e compreende-se que parte destas estavam desenvolvendo atividades laborais em suas casas ${ }^{(6)}$.

Uma das principais preocupações em relação a essa nova realidade é o processo adaptativo do trabalhador em relação ao desenvolvimento de suas funções, tendo em vista que o ambiente, as relações e até mesmo os recursos disponíveis não são os mesmos em relação ao vivenciado anteriormente. Há também que se pensar nos aspectos contraditórios dessa modalidade de trabalho: ao mesmo tempo em que o trabalhador está se protegendo, há a evidente invasão, pelo trabalho, do tempo de não trabalho e da vida familiar. Frente a essa perspectiva, considera-se que é possível associar os elementos de adaptação ao Home Office à Teoria de Adaptação de Callista Roy ${ }^{(7)}$.

A Teoria Adaptativa aponta alguns pressupostos: A pessoa é um ser biopsicossocial; A pessoa está submetida a constantes mudanças devido a interação com o meio; A adaptação da pessoa é condição essencial para uma resposta positiva da interação com o meio; Toda pessoa tem elementos de adaptação (necessidades 
fisiológicas, autoconceito, papel funcional e interdependências) ${ }^{(7-8)}$.

Assim sendo, o objetivo deste artigo é refletir sobre as implicações do trabalho em Home Office na saúde dos indivíduos, sob a perspectiva da Teoria da Adaptação desenvolvida por Callista Roy. A relevância desta discussão pauta-se na necessidade de socialização de perspectivas teóricas que embasem a adaptação do trabalhador a esta nova realidade que foi potencializada pela pandemia do Covid-19.

\section{MÉTODO}

Reflexão teórica-filosófica, baseada na Teoria da Adaptação desenvolvida por Callista Roy. As reflexões e os apontamentos apresentados neste artigo são fruto de discussões realizadas entre os autores, após o expressivo aumento do número de trabalhadores cujas modificações impostas pela pandemia no processo de trabalho culminaram na adoção do trabalho remoto como estratégia viável para a manutenção das atividades laborais.

Para tal, não houve necessidade de apreciação ética, posto que não houve coleta de dados com seres humanos. Ademais, enfatiza-se que a construção da pesquisa procurou apresentar uma análise contemporânea das implicações do trabalho em Home Office, balizada pelos constructos teóricos de Callista Roy.

\section{A TEORIA DA ADAPTACÃO E O HOME OFFICE}

No atual momento de mudanças estruturais no cotidiano laboral brasileiro e de países que enfrentam a pandemia, diversas são as queixas dos trabalhadores que agora se encontram na modalidade Home Office. Portanto, para entender melhor esse contexto social, é necessária uma visão teórica-profissional.

As teorias de enfermagem são concepções articuladas e associadas à realidade, e tem por objetivo descrever, prever e prescrever as práticas de enfermagem ${ }^{(9)}$. A teoria de enfermagem que serviu de inspiração foi o modelo conceitual da adaptação, desenvolvida por Callista Roy, em 1970. Essa teoria ressalta que a enfermagem tem como meta promover a adaptação do indivíduo durante sua doença e saúde, manipulando o ambiente e elementos do paciente ${ }^{(10)}$.

A teórica Callista Roy nasceu em Los Angeles, no ano de 1939, e graduou-se em Enfermagem no Mount St. Mary's College. Em 1966, adquiriu o título de Mestre em Enfermagem Pediátrica pela Universidade da Califórnia em Los Angeles, realizou o curso de Mestrado em Sociologia em 1973, e posteriormente o Doutorado em 1977. A aplicação de sua teoria fundamentou-se no cuidado de enfermagem e em como esse cuidado pode se adaptar à situação de cada indivíduo ${ }^{(11)}$.

A Teoria Adaptativa aponta o ser humano como um indivíduo biopsicossocial, que vive continuamente interagindo com o ambiente ao seu redor, que, por sua vez, está mudando ininterruptamente, criando mecanismos na tentativa de se adaptar; o que pode ou não ocorrer. O processo saúde-doença, então, está intimamente ligado ao resultado que se espera dessa adaptação, que, quando ocorre, é considerada uma resposta positiva aos estímulos internos e externos que o ambiente proporciona ${ }^{(7-9)}$.

Essa teoria possui quatro modos adaptativos que são produzidos ou manifestados, sendo eles: modo fisiológico, modo autoconceito, modo desempenho de papel e modo interdependência. No modo fisiológico a pessoa reage como um ser físico aos estímulos que o ambiente gera, envolvendo cinco necessidades básicas, sendo elas a oxigenação, nutrição, eliminação, atividade e repouso $^{(11)}$.

É importante analisar algumas variáveis envolvidas nessas necessidades básicas. Na nutrição, é imprescindível que o indivíduo tenha uma alimentação balanceada, rica em nutrientes e com as quantidades necessárias. Na modalidade Home Office, em especial na imposição direta pela pandemia de COVID-19, é possível observar dois fenômenos: o esperado e o concreto.

O fenômeno esperado baseou-se na expectativa de redução no custo da alimentação, bem como no incremento na qualidade desta, 
sobretudo pela maior disponibilidade temporal do indivíduo para preparar suas próprias refeições, o que pode ser entendido como um benefício direto ao trabalhador ${ }^{(12)}$. Entretanto, o fenômeno concreto aponta para uma significativa perda de qualidade nutricional, visto que o estresse e a ausência de atividades que proporcionem satisfação para os indivíduos os levam a ingerir alimentos que proporcionem não apenas saciedade, mas também conforto, como é o caso de carboidratos que estimula a produção de serotonina ${ }^{(13)}$.

Ademais, ressalta-se que este novo padrão alimentar vem sendo considerado um fator de risco para o surgimento e agravamento de doenças cardiovasculares, já que alia a má nutrição com práticas sedentárias ${ }^{(14)}$

As variáveis atividade e repouso estão interconectadas quando tratamos do trabalho remoto. No contexto de pandemia, muitos profissionais que agora estão em modalidade Home Office sofrem alterações no seu cotidiano de trabalho, mesclando atividades laborais com horas de descanso, em um eterno looping. Até mesmo o padrão de sono dos trabalhadores foi alterado pela situação de isolamento, quando se observou a manutenção do número de horas, mudanças no horário de início do sono e diminuição da qualidade do sono ${ }^{(15)}$.

Apesar da ubiquidade laboral já ter sido apontada em outros cenários anteriores à pandemia, observa-se que o trabalho remoto é um fator de risco para essa situação ${ }^{(16)}$. O risco supracitado é potencializado quando analisamos sob a perspectiva de gênero. Culturalmente as mulheres são responsáveis por boa parte do trabalho não remunerado dentro de seus lares, utilizando parte do dia no cuidado a crianças e idosos que coabitam a mesma casa, tornando as horas de descanso, uma nova jornada laboral ${ }^{(17)}$.

Cabe ainda ressaltar que a questão financeira, por exemplo, é um fator importante na determinação da qualidade do ambiente em sua totalidade, influenciando na moradia, na alimentação e na sua manutenção. Nesse sentido, as pessoas com melhor condição financeira tendem a possuir um ambiente mais apropriado para a modalidade Home Office e, portanto, fica claro que há uma diferença na realidade baseada na condição social e econômica. Ou seja, é preciso considerar as variáveis de classe social e profissional, de que forma os modos de viver se modificam, e os recursos do trabalhador para gerenciar a produção e reprodução da vida cotidiana.

O modo autoconceito está relacionado aos aspectos psicológicas e espirituais do indivíduo, dimensão na qual este possui liberdade para escolher suas preferências espirituais e outras questões relacionadas à psique humana ${ }^{(8)}$.

É importante frisar que o modo autoconceito é dividido em duas facetas. A primeira é denominada "eu físico" e diz respeito à percepção do indivíduo em relação a todas as suas características físicas $^{(11)}$, o que pode ser prejudicada no contexto de pandemia, já que devido ao isolamento, a ausência de tratamentos estéticos, a nova rotina alimentar, e até mesmo o sedentarismo experimentado por muitos pode modificar a aparência e a percepção corporal.

A segunda faceta está relacionada ao "eu pessoal" e é baseada em três aspectos: a autoconsistência que é a resistência do indivíduo para evitar o desequilíbrio, o autoideal que está relacionado com aquilo que a pessoa espera de si própria e do mundo, e o eu espiritual-ético-moral que está baseado em crenças e valores ${ }^{(11)}$.

Ao trançar o autoconceito com a prática do Home Office, é possível notar que o indivíduo possui maior gestão do seu tempo, tendo a liberdade para se expressar de maneiras diferenciadas do que ocorre presencialmente. Se por um lado, o isolamento pode afetar a percepção física, por outro, imagina-se que este pode ser também uma estratégia fundamental para conexão com sua própria essência, o "eu pessoal”.

No entanto, a flexibilização do trabalho, expressa na modalidade Home Office, quando analisada em profundidade, evidencia que há diferenças importantes entre as categorias e os tipos de trabalho. Em um estudo na França, por exemplo, verificou-se que a flexibilização do horário e do local de trabalho é melhor suportada quando se trata de um trabalho com número limitado de horas na jornada de trabalho, 
porém passa a ser insuportável quando há a simples flexibilização de trabalhos que exigem longas horas ${ }^{(18)}$.

Não se pode negligenciar que o lado psíquico dos indivíduos atuantes em trabalho remoto pode demonstrar fragilidade devido à falta de socialização, mesmo com o aumento da interação familiar no domicílio, o que pode gerar sintomas ansiosos, preocupações exageradas com familiares e amigos e, sobretudo, dúvidas com relação ao futuro ${ }^{(5)}$. No contexto de pandemia, observa-se que muitos indivíduos acreditam em um novo processo de trabalho, no qual o trabalho remoto estará incorporado de modo intrínseco, o que eleva ainda mais o número de incertezas vivenciadas por esses trabalhadores.

Nesse sentido, observa-se que dentre os possíveis acometimentos à saúde dos trabalhadores em trabalho remoto, os adoecimentos mentais/emocionais são aqueles de maior expressão no cenário atual. A respeito disso, observou-se o aumento da prevalência de sintomas ansiosos e depressivos na população durante a quarentena ${ }^{(19)}$, e essa taxa chega a ser o dobro quando comparada à população que não estava vivenciando a quarentena ${ }^{(20)}$.

O modo desempenho de papel se atribui às funções sociais desempenhadas pelo indivíduo, e como este se enxerga inserido no meio social. Em um momento crítico, como o de pandemia, é difícil a inserção física nesse meio, o que pode gerar dificuldades na identificação do papel que é exercido na sociedade ${ }^{(21)}$.

Compreende-se que os papéis desempenhados na sociedade dependem das relações existentes entre os indivíduos, e o distanciamento social pode gerar problemas na compreensão dos papéis. Ora, para que houvesse a figura do empregado era necessária a existência do papel do empregador, contudo, atualmente, a relação laboral ocorre de maneira totalmente desvinculada do sentido lógico presencial ${ }^{(11)}$.

O modo interdependência tem como foco as relações interpessoais e as interações sentimentais ${ }^{(21)}$. Com a recomendação de isolamento social feita pela Organização Mundial da Saúde (OMS), milhões de pessoas deixaram de interagir socialmente com seus familiares e amigos, a fim de evitar a propagação do novo Coronavírus ${ }^{(1)}$, e como produto desse isolamento, um déficit nas relações interpessoais foi gerado, o que pode afetar a saúde mental e física dos indivíduos. Uma solução encontrada para tentar manter essas relações, foi o uso de redes sociais com o auxílio de tecnologias como computadores e smartphones.

Ademais, há de se considerar que o isolamento físico ocorreu por uma situação imposta, ou seja, não fora opção do indivíduo viver e trabalhar com essas particularidades, sofrendo influências do meio em que vive, mas também com vulnerabilidades e deficiências de acesso e manejo das tecnologias existentes como mediação na interação e troca para produção de cada indivíduo no trabalho.

Não obstante, toda fase de mudança na vida dos indivíduos gera uma necessidade ampliada em relação aos sentimentos de afirmação e respeito, que são encontradas de modo natural nas relações sociais físicas ${ }^{(8,11)}$. Assim sendo, ressalta-se a emergência das relações saudáveis, ainda que de maneira virtual, de modo a suprir essa demanda social, visto que para muitos trabalhadores, estas são as únicas interações possíveis no contexto de pandemia.

A enfermagem possui um papel fundamental no acompanhamento dos indivíduos em todas as etapas da Teoria de Callista Roy. No que concerne ao trabalho desenvolvido no contexto de Home Office, observa-se o protagonismo do papel educativo da enfermagem auxiliando no estabelecimento de estratégias que facilitem toda a engrenagem adaptativa.

\section{CONCLUSÃO}

Os profissionais atuantes no trabalho remoto, no contexto de pandemia, estão interpenetrados em uma condição complexa e penosa, como cansaço, dúvidas e expectativas sobre o futuro, o que pode trazer diversas problemáticas para sua saúde, como a ansiedade e o estresse.

Apesar de haver maior gestão do tempo e também aumento do convívio familiar, o 
trabalhador atuante no Home Office possui enorme vulnerabilidade em relação a sua interpretação de seu papel na sociedade, ao atendimento de suas necessidades fisiológicas, a compreensão de suas questões particulares de cunho íntimo e também de suas relações que se tornam diferenciadas com o distanciamento social. Além disso, não se pode deixar de considerar que o Home Office insere-se na modalidade de trabalho flexível, e incorpora mudanças que se alinham com as propostas de um modelo neoliberal de sociedade e economia, ou seja, é preciso que não haja apenas uma adesão ingênua por parte do trabalhador, ainda que imposta por uma situação de crise sanitária, como é a da pandemia.

É necessário observar os contextos relacionados a grupos populacionais específicos, nos quais se observa o maior impacto na saúde e no processo adaptativo, como por exemplo, as mulheres, cuja rotina de trabalho remoto culminou na extensão interminável da jornada de trabalho.

A Teoria Adaptativa de Callista Roy subsidia as discussões sobre a temática e sobre a possibilidade de adaptação nesse novo contexto, seja de maneira pontual ou mediante transformações no processo de trabalho a longo prazo, superando limitações do indivíduo e descobrindo maneiras de se fazer e ser no campo do trabalho.

\section{Colaborações:}

1 - concepção, projeto, análise e interpretação dos dados: Nathalia Henriques Veiga, Yan Zi Li Figueiredo Ten e Vithória Paes Machado;

2 - redação do artigo e revisão crítica relevante do conteúdo intelectual: Nathalia Henriques Veiga, Yan Zi Li Figueiredo Ten, Vithória Paes Machado, Magda Guimarães de Araújo Faria, Mercedes de Oliveira Neto e Helena Maria Scherlowski Leal David;

3 - aprovação final da versão a ser publicada: Nathalia Henriques Veiga, Yan Zi Li Figueiredo Ten, Vithória Paes Machado, Magda Guimarães de Araújo Faria, Mercedes de Oliveira Neto e Helena Maria Scherlowski Leal David.

\section{REFERÊNCIAS}

1. Rafael RDMR, Mercedes Neto, Carvalho MMB, David HMSL, Acioli S, Faria MGA. Epidemiology, public policies and Covid-19 pandemics in Brazil: what can we expect? Rev enferm UERJ. 2020;28:e49570. DOI: http://dx.doi.org/10.12957/ reuerj.2020.49570

2. Huang C, Wang Y, Li X, Ren L, Zhao J, Hu Y, et al. Clinical features of patients infected with 2019 novel coronavirus in Wuhan, China. Lancet. 2020;395:497-506. DOI: https://doi.org/10.1016/ S0140-6736(20)30183-5

3. Pan American Health Organization. WHO Characterizes COVID-19 as a pandemic [Internet]. Washington, D.C. (USA); 2020 [cited 2020 Jun 15]. Available from: https://www.paho.org/hq/index. php?option $=$ com_content $\&$ view $=$ article $\&$ i $\mathrm{d}=15756$ : who-characterizes-covid-19-as-apandemic\&Itemid=1926\&lang $=$ en

4. Brasil. Lei $n^{0}$ 13.979, de 6 de fevereiro de 2020 . Dispõe sobre as medidas para enfrentamento da emergência de saúde pública de importância internacional decorrente do coronavírus responsável pelo surto de 2019. Diário Oficial da União [Internet]. Brasília (DF); 2020 [cited 2020 Aug 17]. Available from: https://www.in.gov.br/en/web/dou/-/lei-n13.979-de-6-de-fevereiro-de-2020-242078735

5. Rafalski JC, Andrade AL. Home-Office: Aspectos Exploratórios do Trabalho a partir de Casa. Temas Psicol. 2015;23(2):431-1. DOI: http://dx.doi.org/ 10.9788/TP2015.2-14

6. InLoco. Mapa brasileiro da COVID-19. Índice de isolamento social [Internet]. Recife; 2020 [cited 2020 Jun 15]. Available from: https:// mapabrasileirodacovid.inloco.com.br/pt/

7. Roy C. Extending the roy adaptation model to meet changing global needs. Nurs Sci Q. 2011;24(4):345-51. DOI: https://doi.org/10.1177\% 2F0894318411419210

8. Roy C, Whetsell MV, Frederickson K. The Roy Adaptation Model and Research. Nurs Sci Q. 2009;22(3):209-11. DOI: https://doi. org/10.1177\%2F0894318409338692

9. Medeiros LP, Souza MBC, Sena JF, Melo MDM, Costa JWS, Costa IKF. Modelo de Adaptação de Roy: revisão integrativa dos estudos realizados à luz da teoria. Rev Rene. 2015;16(1):132-40. DOI: https://doi.org/10.15253/2175-6783.2015000100017 
10. Zavala-Pérez IC, Palacios-Fonseca C, Olea-Gutiérrez CV, Salas-Medina DL, Mercado-Rivas MX. Experiencias adaptativas de mujeres mastectomizadas: una mirada desde el Modelo de Callista Roy. Cult los Cuid Rev Enfermería y Humanidades. 2019;23(53):39-50. DOI: https://doi.org/10.14198/cuid.2019.53.05

11. Batista AH, Santiago MAMT, Matias R de C. Teoria da Adaptação - Callista Roy. In: Braga CG, Silva JV, organizadores. Teorias de Enfermagem. São Paulo: Iátria; 2011.

12. Haubrich DB, Froehlich C. Benefícios e Desafios do "Home Office" em Empresas de Tecnologia da Informação. Rev Gestão Conex. 2020;9(4):167-84. DOI: https://doi.org/10.13071/ regec.2317-5087.2020.9.1.27901.167-184

13. Muscogiuri G, Barrea L, Savastano S, Colao A. Nutritional recommendations for CoVID-19 quarantine. Eur J Clin Nutr. 2020;47:850-1. DOI: https://doi.org/10.1038/s41430-020-0635-2

14. Mattioli AV, Ballerini Puviani M, Nasi M, Farinetti A. COVID-19 pandemic: the effects of quarantine on cardiovascular risk. Eur J Clin Nutr. 2020;74:852-5. DOI: https://doi.org/10.1038/s41430-020-0646-Z

15. Cellini N, Canale N, Mioni G, Costa S. Changes in sleep pattern, sense of time and digital media use during COVID-19 lockdown in Italy. J Sleep Res. 2020;e13074. DOI: https://dx.doi. org/10.1111\%2Fjsr.13074

16. Lara RC, Quartiero EM, Bianchetti L. Trabalho ubíquo na pós-graduação stricto sensu em educação: in/extensificação e multitarefa. Rev Bras Educ. 2019;24:e240014. DOI: http://dx.doi. org/10.1590/S1413-24782019240014
17. Organização das Nações Unidas. Policy Brief: The Impact of COVID-19 on Women [Internet]. New York (EUA); 2020 [cited 2020 Aug 15]. Available from: https://www.un.org/sites/un2. un.org/files/policy_brief_on_covid_impact_on_ women_9_apr_2020_updated.pdf

18. Askenazy P. Shorter work time, hours flexibility, and labor intensification. East Econ J [Internet]. 2004 [cited 2020 Jun 19];30(4):603-14. Available from: https://econpapers.repec.org/scripts/redir. pf?u=http $\% 3 \mathrm{~A} \% 2 \mathrm{~F} \% 2 \mathrm{Fwebholycrossedu} \% 2 \mathrm{FRePEc} \%$ 2Feej\%2FArchive\%2FVolume30\%2FV30N4P603_614. pdf;h=repec:eej:eeconj:v:30:y:2004:i:4:p:603-614

19. Peng M, Mo B, Liu Y, Xu M, Song X, Liu L, et al. Prevalence, risk factors and clinical correlates of depression in quarantined population during the COVID-19 outbreak. J Affect Disord. 2020;275:114-21. DOI: https://dx.doi.org/10.1016\% 2Fj.jad.2020.06.035

20. Lei L, Huang X, Zhang S, Yang J, Yang L, Xu M. Comparison of Prevalence and Associated Factors of Anxiety and Depression among People Affected by versus People Unaffected by Quarantine during the COVID-19 Epidemic in Southwestern China. Med Sci Monit. 2020;26:e924609-1. DOI: https:// dx.doi.org/10.12659\%2FMSM.924609

21. Hamadé DC eleto H, Moraes CS, Costa CCP, Martins MOD. Diagnósticos de enfermagem com pacientes coronariopatas à luz da teoria de Callista Roy. Rev Pesqui. 2020;12:130-7. DOI: https://doi. org/10.9789/2175-5361.rpcfo.v12.7137

Recebido: 29 de junho de 2020

Aprovado: 13 de agosto de 2020

Publicado: 26 de novembro de 2020

A Revista Baiana de Enfermagem utiliza a Licença Creative Commons - Atribuição-NãoComercial 4.0 Internacional. https://creativecommons.org/licenses/by-nc/4.0/

Este artigo é de acesso aberto distribuído sob os termos da Licença Creative Commons (CC BY-NC). Esta licença permite que outros remixem, adaptem e criem a partir do seu trabalho para fins não comerciais. Embora os novos trabalhos tenham de lhe atribuir o devido crédito e não possam ser usados para fins comerciais, os usuários não têm de licenciar esses trabalhos derivados sob os mesmos termos. 